\title{
Evolución de la natación colombiana a través de los juegos olímpicos desde 1948-2017
}

\author{
Evolution of the swimming in Colombia through the \\ Olympic Games from 1948-2017
}

\section{A evolução da natação colombiana através das Olimpíadas desde 1948-2017}

\author{
Sandra Milena Moreno \\ Magister en Metodología del Entrenamiento Deportivo \\ Universidad del Tolima \\ smmorenolv@ut.edu.co
}

\section{Resumen}

Para todo fenómeno social el conocimiento de sus orígenes es un momento imprescindible del análisis y un componente irrenunciable de su explicación. Se describe un recorrido sociohistórico de la evolución de este deporte en Colombia, es preciso reconocer los protagonistas y competiciones que hicieron historia. Por tanto, esta revisión tiene como objeto de estudio describir cómo ha evolucionado la participación de los nadadores colombianos y sus marcas, tomando como referencia los Juegos Olímpicos (JJ. OO) desde 1948- 2017, que se realiza cada cuatro años. Este trabajo, con un marcado de carácter descriptivo, igualmente se realizó la búsqueda en los periódicos de circulación nacional en físico y digitales, memorias de eventos, noticias del comité olímpico colombiano, entrevistas. Se seleccionaron las noticias deportivas publicadas relacionadas con los Juegos olímpicos. Con fundamento en lo anterior, se formula la siguiente pregunta: ¿Cuáles fueron las participaciones, técnica y distancias de nadadores colombianos en los juegos olímpicos?

Palabras claves: Natación, historia, nadadores, Juegos Olímpicos.

\section{Summary}

Knowing the origin of social phenomena is an essential moment of the analysis and a critical component for its explanation. That is the reason why this paper describes the socio-historical timeline of the evolution of the swimming in Colombia. It is necessary to identify the principal actors and the competitions that were a success. One of the main objectives of this study is to describe the way in 
which the participation of the Colombian swimmers in technical, as well as in DISTANCE has evolved, considering as a referent the Olympic Games (JJ. OO) from 1948-2017, which taka place every four years.

Within a descriptive framework search was carried out in the local newspapers by selecting the news related to the Olympic Games. The research question is "How well did Colombian swimmers do (technical y distance) in the Olympic Games?

Keywords: Swimming, history, swimmers, Olympic Games.

\section{Resumo}

Para todo fenómeno social o conhecimento das suas origens é um momento imprescindível de análise e um componente da sua explicação. Nesse artigo se descreve o percurso sócio-histórico da evolução deste esporte na Colômbia reconhecendo que é preciso reconhecer os protagonistas e competições que fizeram história. Para tanto, essa revisão tem por objeto de estudo a descrição de como tem evolucionado a participação dos nadadores colombianos e os seus registros, tendo como referência as Olimpíadas desde 1948 até 2017, realizadas a cada quatro anos. Esse trabalho de caráter descritivo está baseado na pesquisa documental que utilizou jornais nacionais, disponíveis em formato físico e digital, memórias de eventos, notícias do Comité Olímpico Colombiano e entrevistas. Foram escolhidas as matérias esportivas publicadas com relação às Olimpíadas. Tendo isto em consideração, formulou-se a pergunta: quais foram as participações, técnica e distância dos nadadores colombianos nas Olimpíadas no período estudado?

Palavras-chave: Natação, história, nadadores, olimpíadas

Desde el principio de los tiempos el agua ha sido elemento esencial de la vida del hombre, este medio acuático brindó soluciones a muchas necesidades del hombre desde la antigüedad como fueron: la supervivencia, defensa, delimitación de territorio, transporte, comunicación, higiene, alimentación y recreación. El agua es un elemento que está presente en la vida del ser humano desde su nacimiento, y representa del 40 al $60 \%$ de su peso corporal (McArdle, Katch y Katch, 1990).

La natación es "la acción y efecto de nadar" y nadar es el hecho de trasladarse en el agua, ayudándose de los movimientos necesarios, y sin tocar el suelo ni otro apoyo (Real Academia Española, 1997), por otra parte, aparecen términos como energías: "Avance voluntario en un líquido elemento, merced a las propias energías" (Iguarán, 1972), o incluso el término sostenerse: "Medio que permite al hombre sostenerse y avanzar en el agua" (Rodríguez,
1997). Basándonos en Arellano (1992), la natación es

la habilidad acuática que permite al ser humano desplazarse en un medio líquido, gracias a las fuerzas propulsivas que genera con los movimientos de los miembros superiores, inferiores y cuerpo, que le permiten vencer las resistencias que se oponen al avance.

Es necesario recalcar que la definición es también orientada de acuerdo con los objetivos de su práctica, desde lo más sencillo la supervivencia, enseñanza de la técnica, higiene, terapéutica, hasta lo recreativo y competitivo.

La natación competitiva es un deporte olímpico, atlético, acuático, formal, individual, simétrico, asimétrico, cíclico, de tiempo marca, de genero mixto según la prueba (Moreno. S. 2018) 
Una vez definida la natación, al añadirle el adjetivo "deportivo", se obtendría una actividad en la que el ser humano practica un deporte olímpico reglamentado, con el objetivo de desplazarse de la forma más rápida posible en el agua. En esta investigación se pretende recuperar y reconstruir de la narrativa histórica de la natación en Colombia. Es necesario conocer qué ha ocurrido en las últimas décadas. Resulta imprescindible reconocer los protagonistas y competiciones que hicieron historia.

El abordar la participación de la natación colombiana implica establecer periodos cronológicos identificados por acontecimientos y desarrollos sucedidos durante este periodo. Dando importancia al reconocimiento social, cultural y deportivo. Por esto, la reseña comprende desde el año 1948 hasta nuestros días, surgiendo algunos interrogantes, tales como: ¿Cuál fue la primera participación en los Juegos Olímpicos de nadadores colombianos? ¿Quiénes fueron los nadadores que fueron protagonistas en la historia de nuestra natación? ¿Qué técnicas y distancias participaron los nadadores colombianos?

\section{Historia de la natación internacional y nacional}

El origen de la natación es ancestral y se tiene prueba de ello a través del estudio de las más antiguas civilizaciones. El dominio del desplazamiento en el agua forma parte de la adaptación humana desde que los primeros hombres.

Los primeros registros históricos que hacen referencia a la natación aparecen en Egipto en el año 5.000 a.C.'; entre los egipcios el arte de nadar era uno de los aspectos más elementales de la educación pública, así como el conocimiento de los beneficios terapéuticos del agua, lo cual quedó reflejado en algunos jeroglíficos que datan del 2.500 antes de Cristo. Pinturas de la Roca de Gilf Kebir (Lewillie, 1983). Pero el esplendor de Grecia, la natación no se va a desprender de esa mera función de supervivencia; es entonces cuando la natación pasa a ser una parte más de la educación de los griegos, incluso el saber nadar proporcionaba una cierta distinción social ya que cuando se quería llamar inculto o analfabeto a alguien se le decía "no sabe ni nadar ni leer". También se utilizaba como táctica militar (Lewin, 1979; Rodríguez, 1997). Pero saber nadar no se limita a las antiguas Grecia y Roma, sino que se conserva aún en nuestros días.

La natación sí tiene una gran importancia en el entrenamiento militar y como medida recuperadora para los atletas (Jardí, 1996). Al igual que en Grecia, en Roma la natación forma parte de la educación de los romanos, existiendo una visión más recreativa del agua, como fueron las termas romanas que eran recintos públicos destinados a baños típicos de la civilización romana (Nack y Wägner 1966), existían piscinas de más de 70 metros de longitud (Lewin, 1983).

Durante la Edad Media el interés por la natación decrece en gran medida, debido, sobre todo, a la poca atención que se muestra a todo lo relacionado con el cuerpo humano. Sólo en los países del norte de Europa se ve como una actividad beneficiosa (Lewin, 1979; Reyes, 1998).

En el Renacimiento, la práctica de la natación vuelve a resurgir del período de oscurantismo al que estuvo sometida durante la Edad Media y se la considera como una materia idónea dentro de las actividades físicas (Reyes, 1998). Como fruto de esta concepción, surgen los primeros escritos referentes a la natación, según el libro del Alemán Nicholas Wymman (1538) titulado "Colymbetes, Sive de arti natandis dialogus et festivus et iucundus lectu", cuya traducción libre es "El nadador o el arte de nadar, un diálogo festivo y divertido de leer" (Iguarán, 1972).

Es en el siglo XIX, en Inglaterra, la natación alcanza su mayor apogeo. En el año 1828 se construye en Londres la primera piscina cubierta y en el año 1837 se lleva a cabo la primera competición organizada (Reyes, 1998). 
$\mathrm{Al}$ aparecer las primeras competiciones, surge la necesidad de reglarlas; con ese objetivo nace en Inglaterra, en el año 1874, la primera federación de clubes que lleva por nombre "Association Metropolitan Swimming Club", que redacta el primer reglamento de natación, dándose la posibilidad de establecer récord del mundo (Rodríguez, 1997).

También es durante este siglo, en el año 1875, cuando el ser humano cruza a nado por primera vez el Canal de La Mancha, lo hace Matthew Web, quien establece un tiempo de $21 \mathrm{~h}$ y 45 min.

Este deporte nunca formó parte de los Juegos Olímpicos antiguos, pero se presenta en los primeros juegos modernos de Atenas 1896, en 1908 se organizó la Fédération International de Natation Amateur (FINA) para poder celebrar carreras de aficionados. La competición femenina se incluyó por primera vez en los Juegos Olímpicos de 1912.

Originalmente, las pruebas de natación se realizaban en el mar o un lago artificial. En 1908 las pruebas pasaron a realizarse en piscina. En 1924 se incorporaron los carriles, así como la longitud olímpica de 50 metros. El estilo mariposa era permitido en las pruebas de pecho, hasta que se crearon eventos independientes en 1956. Las primeras lentes se permitieron en 1976.

En Colombia el Comité Olímpico fue creado en 1936 y la Federación Colombiana de Natación, FECNA, en agosto de 1939 en Cali.

Colombia tuvo su primera participación de natación en Juegos Olímpicos de 1948 en Londres con los nadadores Luis Child y Luis Eduardo Tiburón González (200 metros estilo pecho). (NadarMas, 2008; Comité Olímpico Colombiano, 2016; Gutiérrez Bueno, 2014).

En los siguientes JJ. OO., celebrados en Melbourne Australia 1956, Colombia participó con los nadadores: Gilberto Martínez, Sergio Martínez y Álvaro Gómez. (Comité Olímpico
Colombiano, 2016)

En los siguientes JJ. OO. Roma 1960, Colombia no tuvo participación en natación carreras.

En los siguientes JJ. OO. celebrados en Tokio 1964, Colombia participó con el nadador Julio Arango, en los 400 metros y libres $1.500 \mathrm{~m}$ libres, no obtuvo tiempo clasificatorio porque ocupó el decimoquinto lugar, con tiempo de 17.59.1, que en ese momento se convirtió en nuevo registro suramericano. sin embargo, se convirtió en ser el nadador más joven con 14 años. (Comité Olímpico Colombiano, 2016; Ávila Palacios, 2016).

En los JJ. OO. de México D.F. (México) en 1968, Participaron por Colombia los nadadores en relevos 4x 100 libre, J. Arango (56.0), Tomás Becerra (59.7), Federico Sicard (58.8), Ricardo González (57.0); el tiempo total: 3:51.5 y los relevos 4 x 200 Relevo Libre: Becerra (2:07.0), Sicard (2:10.1), González (2:07.9) y Arango (2:01.7) para un total de 8:26.7, lograron avanzar hasta la segunda ronda; la primera gran figura fue la vallecaucana Olga Lucía de Angulo, dejándole a Colombia una rica historia deportiva con medallas en diferentes eventos internacionales y la presencia olímpica (NadarMas, 2008).

En los JJ. OO. celebrados en Munich (Alemania) en 1972, la colombiana Roselina Ángel, nadadora paisa, ocupó el lugar 13 en los 800m libre, con un tiempo de 9:38.08 (NadarMas, 2008). En este evento ven al que puede ser considerado el mejor nadador de todos los tiempos, el americano Mark Sptiz, que consigue colgarse siete medallas de oro, cuatro en pruebas individuales (100 m libres; $200 \mathrm{~m}$ libres; $100 \mathrm{~m}$ mariposa; $200 \mathrm{~m}$ mariposa) y tres formando parte del equipo de relevos de EE. UU. Todas estas medallas son conseguidas batiendo los récords del mundo.

En Montreal (Canadá) se celebran los JJ.OO. en 1976, registrándose un total de veinte nuevos 
records del mundo, destacando el del americano Jim Montgomery que se convierte en el primer nadador que consigue nadar por debajo de $50 \mathrm{~s}$ en la prueba de $100 \mathrm{~m}$ libres, al realizar un registro de 49" 99. Hay que destacar también a la alemana democrática Kornelia Hender que consigue cuatro medallas de oro y otros tantos récords del mundo. Asimismo, se instauran por primera vez los controles antidopaje. Participaron los colombianos, el gran nadador tolimense, Helmut Levy fue 15 en los 200 pecho y $100 \mathrm{~m}$ libre; el mariposista Jorge Jaramillo fue puesto 11 en los 200 M y la bogotana María Fernanda Pérez también entró entre los 20 mejores de los Juegos cuando ocupó el puesto 17 en los 800 libre (NadarMas, 2008). En los años siguientes destacan dos figuras de gran relevancia, el ruso Vladimir Salnikov, primer hombre en bajar de los quince minutos en los $1.500 \mathrm{~m}$ libres y la americana Mary T. Meagher que, con un tiempo de 2'05"96 en la prueba de $200 \mathrm{~m}$ mariposa, ostenta el récord mundial durante veinte años, hasta 2000 .

En 1980 se disputan JJ.OO. en Moscú (URSS), que están marcados por el boicot político de algunos de los países occidentales con los EE. UU. a la cabeza. Pablo Restrepo se clasificó para la final olímpica de los 100 metros pecho, logrando el séptimo lugar, superó cuatro años más tarde en Los Ángeles-1984, cuando fue sexto en los 200 metros pecho, resultados que repitió en Seúl-1988 (Colprensa, 2014). El boicot político se repite, cuatro años más tarde, en los JJ.OO. de Los Ángeles (EE. UU.) esta vez son los países socialistas, con la URSS como abanderada de esta postura, los que no asisten a la cita olímpica. Se batieron diez records del mundo, y se dio un hecho que no se repite nunca más en la historia de los JJ. OO., y es el empate por la medalla de oro entre las nadadoras americanas Stenseifer y Hogshead en la prueba de $100 \mathrm{~m}$ libres (Saavedra, Escalante y Rodríguez, 2003).

Dos años después se celebran en Seúl (Corea del Sur) los JJ. OO. 1988, el colombiano Pablo Restrepo hizo su participación $100 \mathrm{~m}$ pecho. La nadadora de la República Democrática Alemana
Kristin Otto, que alcanza seis medallas de oro, el mayor número de medallas de oro tras Mark Spitz, en el 1972.

En 1992, se celebran los JJ. OO. Barcelona, con la participación de Alejandro Bermúdez, no se encontró ninguna referencia en distancia y tiempos.

En 1996, se celebran los JJ. OO. en Atlanta (EE. UU.), en el que los resultados no fueron los mejores, estableciéndose sólo cuatro récords del mundo. La nadadora más destacada es la irlandesa, hasta ese momento desconocida, Michelle Smith, que vence en los 200 y $400 \mathrm{~m}$ estilos y en los $400 \mathrm{~m}$ libres (Rodríguez, 1997). En los Campeonatos de Europa celebrados en Sevilla en 1997, la participación de Alejandro Bermúdez Tamayo nadador colombiano. Compitió en tres Juegos Olímpicos consecutivos desde Barcelona 1992. $\mathrm{Su}$ mejor resultado en estos juegos fue el lugar 13, quinto en la final B, de los $400 \mathrm{~m}$ combinados en Atlanta 1996 con un tiempo de 4:26.64. Bermúdez posee el récord de más medallas de oro en los Juegos Deportivos Nacionales, con un total de 27 en tres ediciones. Es también el nadador colombiano con más marcas nacionales registradas con un total de 94. Fue el primer colombiano en llegar a una final de un Campeonato Mundial de Natación en Piscina Corta, lo hizo en 1995 en Río de Janeiro (NadarMas, 2008), como también la participación de Isabel Ceballos, Armando Serrano, Diego Perdomo, Mauricio Moreno (Comité Olímpico Colombiano, 2016).

En los últimos JJ. OO. celebrados en el 2000, en Sydney (Australia), destacan el gran número de récord batidos, con la supremacía de los holandeses en la prueba de $100 \mathrm{~m}$. libres, teniendo como vencedores, con segundo récord del mundo, a Peter Van de Hoogenband e Ingrid de Bruijn. Destaca también el joven australiano, Ian Thorpe, ganador de los $400 \mathrm{~m}$ libres. La participación del colombiano Camilo José Becerra Velasco de la modalidad de estilo libre, representando a Colombia en los 
Juegos Olímpicos de 2000, 2004 y 2008. La participante colombiana en Atenas-2004, Paola Duguet Duran, nadadora, especialista en las pruebas de fondo de estilo libre y en las pruebas de $400 \mathrm{~m}$ y $800 \mathrm{~m}$ libres. Y en los últimos años surgieron las dos últimas grandes figuras de la natación colombiana: Ómar Andrés Pinzón y Carolina Colorado. Pinzón ganó en 2011 dos medallas de oro en la Copa Mundo Fina, durante la sexta y séptima paradas en la prueba de los 200 metros espalda. Carolina, además de múltiples títulos internacionales, que la llevan con Ciclo Olímpico dorado rumbo a Rio-2016, acumula seis medallas en su carrera deportiva en paradas de Copas Mundo, siendo la única mujer de la historia colombiana en ganar medallas en este tipo de eventos (Colprensa, 2014; El Colombiano, 2004; Revista semana, 2004)

En los JJ. OO. celebrados en Beijing 2008, se contó con la participación de los siguientes nadadores colombianos: Camilo Becerra, Omar Pinzón, Julio Galofre, Carolina Colorado, Erika Stewart (NadarMas, 2008).

En los JJ. OO. Celebrados en Londres, 2012, participaron los nadadores colombianos Carolina Colorado, Mateo De Angulo, Omar Pinzón. Clavados: Víctor Ortega, Sebastián Villa. Desafortunada-mente fueron eliminados
(Revista Semana, 2012; El Espectador, 2012; Colprensa, 2012)

En los JJ. OO., celebrados Río de Janeiro, 2016, Colombia participó con los siguientes nadadores: Isabella Arcila, Jonathan Gómez N, Omar Pinzón, Jorge Murillo (El Espectador, 2016; Comité Olímpico Colombiano, 2016; Revista Semana, 2016)

Isabella desde los 12 años hace parte de la Selección Colombia y desde los 13 está compitiendo a nivel internacional. En el 2010 inició su sueño, tras participar en los Juegos Olímpicos de la Juventud en Singapur en el 2010. Hoy es una de las figuras de la delegación Del Valle del Cauca, los campeones de los XXI Juegos Nacionales Bolívar 2019. Actualmente vive en Estados Unidos donde entrena. Sobre la posibilidad de ser medallista olímpica, Arcila no escatima en dar el 100\% para lograrlo.

Una medalla en Tokio sería una hazaña. Quiero ser la primera colombiana en una final olímpica en natación. Después de estar clasificada entre las 8 mejores del mundo, la competencia está para cualquiera y espero que todos los colombianos me acompañen en Tokio para celebrar juntos. (Ministerio del Deporte, 2019)

\section{Juegos Olímpicos y nadadores colombianos}

\begin{tabular}{|c|c|c|c|c|}
\hline JJ. OO. & Nadador & Técnica & Distancia & Tiempo \\
\hline Londres 1948 & $\begin{array}{l}\text { Luis González } \\
\text { Luis Child }\end{array}$ & Libre & $1500 \mathrm{~m}$ & $20: 40.6$ \\
\hline Melbourne, & Gilberto Martínez, & Libre & $400 \mathrm{~m}$ & 4.38 .08 \\
\hline Australia 1956 & Sergio Martínez & Libre & $100 \mathrm{~m}$ & \\
\hline Roma 1960 & No hubo participación en natación & carreras & & \\
\hline Tokio 1964 & Julio Arango & Libre & $1500 \mathrm{~m}$ & $17: 59.1$ \\
\hline \multirow[t]{4}{*}{ México 1968} & J. Arango (56.0), T. Becerra & & & \\
\hline & (59.7), F. Sicard (58.8), R. & Relevos & $4 \times 100$ & $3: 51.5$ \\
\hline & Gonzalez (57.0) & & $400 \mathrm{~m}$ & $17: 53.9$ \\
\hline & $\begin{array}{l}\text { Becerra (2:07.0), Sicard } \\
(2: 10.1) \text {, Gonzalez }(2: 07.9) \text { y } \\
\text { Arango }(2: 01.7)\end{array}$ & Relevos & $\begin{array}{l}4 \times 200 \\
\text { libre }\end{array}$ & $8: 26.7$ \\
\hline
\end{tabular}




\begin{tabular}{|c|c|c|c|c|}
\hline & Olga L, De Angulo, & Libre & $400 \mathrm{~m}$ & 5:08.06 \\
\hline & Olga L De Angulo & Libre & $400 \mathrm{~m}$ & $4: 40.09$ \\
\hline & Roselina Ángel & Libre & $800 \mathrm{~m}$ & $9: 38.08$ \\
\hline & Jorge Jaramillo & Mariposa & $200 \mathrm{~m}$ & $2: 04.30$ \\
\hline & Helmut Levy & Pecho & $200 \mathrm{~m}$ & $2: 27.94$ \\
\hline & & Libre & $100 \mathrm{~m}$ & 54.60 \\
\hline & María F Pérez & Libre & $800 \mathrm{~m}$ & $9: 36.10$ \\
\hline Moscú 1980 & Helmut Levy. & Pecho & $100 \mathrm{~m}$ & 1:07.06 \\
\hline & & & $200 \mathrm{~m}$ & $2: 27.94$ \\
\hline & Pablo Restrepo & Pecho & $100 \mathrm{~m}$ & $1 ; 05.38$ \\
\hline $\begin{array}{l}\text { Los Ángeles } \\
\mathbf{1 9 8 4}\end{array}$ & Pablo Restrepo & Pecho & $200 \mathrm{~m}$ & $2: 18.96$ \\
\hline Seúl, 1988 & Pablo Restrepo & Pecho & $100 \mathrm{~m}$ & 1.05 .38 \\
\hline Atlanta 1996 & Alejandro Bermúdez & Combinado & $400 \mathrm{~m}$ & $4: 26.64$ \\
\hline & Isabel Ceballos & Libres & $100 \mathrm{~m}$ & \\
\hline & & Pecho & $200 \mathrm{~m}$ & \\
\hline & Armando Serrano & Libre & $200 \mathrm{~m}$ & \\
\hline & Diego Perdomo & Mariposa & $100 \mathrm{~m}$ & \\
\hline & Mauricio Moreno & Libre & $200 \mathrm{~m}$ & \\
\hline Sydney 2000 & Alejandro Bermúdez & Combinado & $400 \mathrm{~m}$ & \\
\hline & & Espalda & $200 \mathrm{~m}$ & \\
\hline & Camilo Becerra & Libre & $50 \mathrm{~m}$ & \\
\hline & Fernando Jácome & Libre & $100 \mathrm{~m}$ & 52.24 \\
\hline & & & $200 \mathrm{~m}$ & $1: 54.17$ \\
\hline & Germán Martínez & Espalda & $100 \mathrm{~m}$ & 52.24 \\
\hline Atenas $\mathbf{2 0 0 4}$ & Camilo Becerra & Libre & $50 \mathrm{~m}$ & \\
\hline & Omar Pinzón & Espalda & $200 \mathrm{~m}$ & $2: 06.20$ \\
\hline & Paola Duguet & Libres & $400 \mathrm{~m} \mathrm{y}$ & $4: 20.69$ \\
\hline & & & $800 \mathrm{~m}$ & $9: 06.96$ \\
\hline Beijing 2008 & Camilo Becerra & Libre & $50 \mathrm{~m}$ & \\
\hline & Omar Pinzón & & $100 \mathrm{~m}$ & 55.11 \\
\hline & & Espalda, & $200 \mathrm{~m}$ & $1: 59.86$ \\
\hline & & Espalda & $200 \mathrm{~m}$ & $2: 02.28$ \\
\hline & & Combi & $400 \mathrm{~m}$ & $4: 22.31$ \\
\hline & & Indv & & \\
\hline & & Combi & $200 \mathrm{~m}$ & $1: 59.47$ \\
\hline & & Indv & & \\
\hline & & Mariposa. & & \\
\hline & Julio Galofre & libre. & $200 \mathrm{~m}$ & $1: 50.62$ \\
\hline & Carolina Colorado & Libre & $50 \mathrm{~m}$ & 26.11 \\
\hline & & Espalda & $100 \mathrm{~m}$ & 1:01.19 \\
\hline & & Mariposa & $100 \mathrm{~m}$ & $1: 00.06$ \\
\hline & Erika Stewart & Combi & $200 \mathrm{~m}$ & $2: 18.54$ \\
\hline & & Indiv & & \\
\hline Londres 2012 & Carolina Colorado & Espalda & $100 \mathrm{~m}$ & $1: 01.81$ \\
\hline & & & $200 \mathrm{~m}$ & $2: 13.64$ \\
\hline & Mateo De Angulo & Libre & $400 \mathrm{~m}$ & $3: 57: 76$ \\
\hline & Omar Pinzón & Espalda & $100-200$ & $1: 58.99$ \\
\hline & & Mariposa & $200 \mathrm{~m}$ & \\
\hline
\end{tabular}




\begin{tabular}{lllll} 
Río de Janeiro & Isabela Arcila & Libre & $50 \mathrm{~m}$ & 25.52 \\
$\mathbf{2 0 1 6}$ & & & & \\
& Jonathan Gómez & Mariposa & $200 \mathrm{~m}$ & $1: 56.65$ \\
& Omar Pinzón, & Espalda & $200 \mathrm{~m}$ & $1: 59.69$, \\
& Jorge Murillo & Pecho & $100 \mathrm{~m}$ & $1: 00.81$ \\
& & $200 \mathrm{~m}$ & $2: 18.81$ \\
\hline
\end{tabular}

Tabla 1. Nadadores colombianos en los Juegos Olímpicos. Elaborado por la autora.

\section{Conclusiones}

Sin duda alguna, fueron los Juegos Olímpicos de la Era Moderna los cuales dieron el impulso definitivo para que fueran apareciendo y consolidándose las diferentes técnicas de nado que conocemos hoy en día.

Así, en Atenas (1896) sólo existió la técnica libre. En París (1900) se ejecutó la técnica libre y espalda. En 1912, el americano Habner realiza el mismo tipo de nado, pero utilizando un movimiento de los miembros inferiores en forma de pedaleo, dicho estilo fue denominado Espaldacrol (Rodríguez, 1997). En San Luis (1904) se añadió la braza y en Melbourne-1956 la braza-mariposa, produciéndose su separación definitiva en 1953 (Reyes, 1998).
Las últimas innovaciones hacen referencia a cuestiones de material y equipamiento deportivo, como el taco o partidores, piscinas de profundidad óptima, vestidos de baño etc. Pero todo esto excede los propósitos del presente artículo.

La primera participación del país en los Juegos Olímpicos fue en Berlín en 1932, de manera extraoficial. La natación colombiana inicia oficialmente en los juegos olímpicos desde 1936 de allí ha venido participando en diversas competencias internacionales y nacionales, por lo que cuatro años después se fundó el Comité Olímpico Colombiano (COC) y la Federación Nacional de Natación. Colombia ha tenido una

\begin{tabular}{llllll}
\hline NADADOR & TECNICA & \multicolumn{2}{l}{ DISTANCIA TIEMPO } & JJ.OO. ORO & $\begin{array}{l}\text { PAIS GANADOR } \\
\text { DEL ORO }\end{array}$ \\
\hline $\begin{array}{l}\text { Isabela } \\
\begin{array}{l}\text { Arcila } \\
\text { Jonathan }\end{array}\end{array}$ & Libre & $50 \mathrm{~m}$ & 25.52 & 24.07 & DINAMARCA \\
$\begin{array}{l}\text { Gómez } \\
\text { Omar }\end{array}$ & Espalda & $200 \mathrm{~m}$ & $1: 59.69$, & 1.53 .62 & EE.UU \\
$\begin{array}{l}\text { Pinzón } \\
\text { Jorge }\end{array}$ & Pecho & $100 \mathrm{~m}$ & $1: 00.81$ & 52.13 & REINO UNIDO \\
Murillo & & $200 \mathrm{~m}$ & $2: 18.81$ & 2.07 .43 & KAZAJISTAN \\
\hline
\end{tabular}

Tabla 2. Marcas comparativas de participación y medalla de oro en los JJ. OO. Río de Janeiro, 2016. Elborado por la autora.

muy buena participación en los Juegos Olímpicos, pero no ha obtenido ninguna medalla en los juegos olímpicos en este deporte en la modalidad carreras.

Asimismo, de las 49 participaciones que Colombia ha tenido, el mayor porcentaje se lo lleva la técnica Libre con un $42.8 \%$, seguido por la técnica de espalda con un $18.3 \%$, Pecho y la prueba de relevos con un empate del $14.2 \%$ y en menor participación la técnica Mariposa con un $10.2 \%$. En cuanto a las distancias, la que mayor participación tiene con un $35 \%$ fue $200 \mathrm{~m}$, seguido del $26.7 \%$ con $100 \mathrm{~m}$, un $16 \%$ para $400 \mathrm{~m}$ y un $5.3 \%$ para $800 \mathrm{~m}$. 
En la Tabla 2 se puede observar los tiempos que realizaron por cada prueba y las marcas ganadoras de medalla de oro en los juegos olímpicos de Rio 2016, las cuales algunas están muy alejadas y en otras en tan solo segundos. Cabe aclarar que en este deporte es difícil bajar segundos. De hecho, también se puede evidenciar la participación masculina en un $72.7 \%$ mientras que la femenina estuvo en un $27.2 \%$, lo que nos indica que la natación de alto rendimiento es más predominante el género masculino atener altos logros deportivos.

Considerando que es de interés este articulo para la comunidad que gusta de este deporte, se puede deducir actualmente por los entrenadores, nadadores y dirigentes de en Colombia carecen de recursos materiales, humanos, proyectos de capacitación, actualización y planes de formación de nadadores a futuro; falta unificar conceptos, metodologías, un sistema de preparación para los futuros nadadores y sobretodo no hay apoyo en cuanto al mejoramiento de infraestructura y construcción de nuevos escenarios.

Desafortunadamente por lo anterior el poco apoyo a los nadadores hacen que la tasa de deserción de deportistas de alto rendimiento sea elevada, así mismo hay aumento de migración a ligas de mayor potencia, así mismo alternar los estudios académicos con los entrenamientos deportivos es complejo y sin apoyo aún más.

Al igual que lo mencionado anteriormente, algunos entrenadores pretenden trabajar presentando planes de entrenamiento en formato de "recetario" aplicando lo que a unos les ha servido, cuando uno de los principios es la individualidad. Existen todavía demasiadas áreas en las que predominan las preguntas en lugar de las respuestas, pudiendo sacar y desarrollar sus propios planes innovadores de entrenamiento. Por lo que la federación debería ofrecer capacitaciones o clínicas en convenio de otros países para avanzar en cuanto en la formación de entrenadores e instructores desde los más jóvenes que son la base de este deporte. Por lo tanto, la alternativa más favorable es: capacitación a entrenadores y formadores, estímulos, becas y apoyos económicos, un sistema de selección de talentos que permita alcanzar altos resultados es necesario: la detección, selección y seguimiento de los nadadores de formación que presentan capacidades y aptitudes, para sean sometidos a un proceso de preparación donde puedan ser transferidos al alto rendimiento respetando sus etapas de desarrollo biológicas, haciendo uso de avances científicos de las diferentes ramas de las ciencias aplicadas y de la tecnología actual. Inversión en infraestructuras, centro de valoración de alto rendimiento a futuro.

Ser nadador de alto rendimiento implica esfuerzo, disciplina y compromiso. Pero uno de los componentes esenciales es la pasión. Sin ella no es posible alcanzar ninguna meta. El nadador olímpico nace y a lo largo de su vida se entrena.

\section{Referencias}

Ávila Palacios, R. (2016, 16 de agosto). Colombia en los Juegos Olímpicos: 84 años de una historia apasionante. El Espectador. www.elespectador.com/deportes/otrosdeport es/colombia-los-juegosolimpicos-80-anos-de-u na-historia-a-articulo-648888

Colprensa. (2012, 29 de julio). Carolina Colorado y Omar Pinzón, eliminados en los 100 metros espalda. El Colombiano.

https://www.elcolombiano.com/historico/car olina_colorado_y_omar_pinzon_fueron_elimina dos_en_los_100_metros_espalda-IUEC_199573

Colprensa. (2014, 14 de octubre) La natación colombiana y sus 75 años de crecimiento deportivo. Vanguardia. https://www.vanguardia.com/deportes/otro s-deportes/la-natacion-colombiana-y-sus-75anos-de-crecimiento-deportivo-PQVL282827

Comité Olímpico Colombiano. (2016, 19 de julio). Colombia en los Olímpicos (2): Londres 1948.

http://www.coc.org.co/all-news/colombia-en 
-los-olimpicos-2-londres-1948/

Comité Olímpico Colombiano. (2016, 24 de julio). Colombia en los Olímpicos (5): Tokio 1964.

http://www.coc.org.co/all-news/colombia-en -los-olimpicos-5-tokio-1964/.

Comité Olímpico Colombiano (2016, 29 de julio). Historial natación colombiana en los Juegos Olímpicos.

http://www.coc.org.co/all-news/historial-nat acion-colombiana-en-lo-juegos-olimpicos/

FECNA. (2013). Fecna Federación Colombiana De Natación. Recuperado el 18 de marzo de 2014, de: http://www.fecna.com/

FINA. (2014). Fina.org. Recuperado el 18 de marzo de 2014, de: http://www.fina.org/

Gutiérrez Bueno, L. (2014, 14 de noviembre). Luis González, el "Tiburón” que se volvió una leyenda. El País.

https://www.elpais.com.co/deportes/luis-go nzalez-el-tiburon-que-se-volvio-una-leyenda. html

Iguarán, J. (1972). Historia de la natación antigua y de la moderna de los juegos olímpicos.

Jardí, C. (1996). Movernos en el agua. Barcelona: Paidotribo. La Doce: Odecabe se reunirá en febrero en Barranquilla para evaluar los Juegos Centroamericanos 2018

Jonathan Gómez: a semifinal de natación y sin miedo a Phelps. (2016, 8 de agosto). Revista Semana. https://www.semana.com/juegos-olimpicos-r io-2016-jonathan-gomez-en-semifinal-natacio n-200-metros-mariposa/485196/

Las figuras que estarán presentes en los Juegos. (2004) El Colombiano. https://www.elcolombiano.com/historico/z figurasjuegos_colprensa_jlopa_25112004-HVE
C_AO_4005662

Lewillie, L. (1983). Research in swimming: historical and scientific aspects. A. Hollander, P. Huijing y D. Groot (Eds.), Biomechanics and Medicine in Swimming IV (pp. 7-16). Champaign: Human Kinetics.

Lewin, G. (1979). Natación. Madrid: Augusto Pila Teleña.

Lewin, G. (1983). Natación. Madrid: Pila Teleña.

Lista delegación colombiana para los Juegos Olímpicos. (2004, 8 de agosto). Revista Semana.

https://www.semana.com/noticias/articulo/ lista-delegacion-colombiana-para-juegos-olim picos/67450-3/

Mateo de Angulo: Aún estamos en otro nivel. (2012, 2 de agosto). El Espectador. https://www.elespectador.com/deportes/ma teo-de-angulo-aun-estamos-en-otro-nivel/.

McArdle, W., Katch, F. y Katch, V. (1990). Fisiología del ejercicio. Madrid, España: Alianza Editorial.

Ministerio del Deporte. (2019, 1 de diciembre). Isabella Arcila, figura de la natación colombiana.

https://www.mindeporte.gov.co/index.php? idcategoria $=96924$

Moreno. S. (2018) Historia de la natación en el Tolima, a partir de los clubes, piscinas y competiciones 1965-2018. Ibagué, Colombia: Universidad del Tolima.

Nadador Omar Pinzón, tras las huellas del mítico Pablo Restrepo. (2012, 23 de julio). Revista Semana.

https://www.semana.com/nadador-omar-pin zon-tras-huellas-del-mitico-pablo-restrepo/ 26 1682-3/ 
NadarMas. (2008, 7 de agosto). La natación de Colombia en los Juegos Olímpicos. http://www.nadarmas.com/?p=645

Nack, E. y Wägner W. (1966.) ROMA. El país y el pueblo de los antiguos romanos. Editorial Labor, S. A.

Real Academia Española. (1997). Diccionario de la Lengua Española (21 ${ }^{\mathrm{a}}$ ed.). Madrid, España: Espasa Calpe.

Reyes, R. (1998). Evolución de la natación española a través de los campeonatos de natación de invierno y verano desde 1977 a 1996. Tesis Doctoral. Universidad de Las Palmas de Gran Canaria.

Rodríguez, L. (1997). Historia de la natación y evolución de los estilos. Natación, Saltos y Waterpolo, 19 (1),38-49.

Saavedra, J., Escalante, Y. y Rodríguez, F. (2003) La evolución de la natación. Revista Digital, (66).

https://www.efdeportes.com/efd66/natacion. htm 